\title{
Low Prevalence of Celiac Disease among Patients with Functional Gastrointestinal Disorders in Latvia
}

\author{
Alise Dekante $^{1^{\star}}$, Ilva Daugule ${ }^{1,3}$, Sergejs Pavlovics ${ }^{1}$, Ilze Kikuste ${ }^{1,2,3}$, Inese Polaka ${ }^{3}$, Lilian Tzivian ${ }^{1,3}$, Ilona Kojalo $^{3}$, \\ Viesturs Putnins $^{4}$, Ivars Tolmanis ${ }^{2}$, Ingrida Rumba-Rozenfelde ${ }^{1}$, Sarmite Boka ${ }^{1}$, Aigars Vanags ${ }^{2,3}$, Marcis Leja ${ }^{1,2,3}$
}

\author{
1) Faculty of Medicine, \\ University of Latvia, Riga, \\ Latvia \\ 2) Digestive Diseases Centre \\ GASTRO, Riga, Latvia \\ 3) Institute of Clinical \\ and Preventive Medicine, \\ University of Latvia, Riga, \\ Latvia \\ 4) Academical Laboratory of \\ Histology, Riga, Latvia
}

\begin{abstract}
Background \& Aims: Studies suggest that the prevalence of celiac disease (CD) is increased in individuals with functional gastrointestinal disorders (FGIDs), in particular, irritable bowel syndrome (IBS); however, the evidence is conflicting. We aimed to analyze the prevalence of CD in patients with FGIDs in Latvia. Methods: This retrospective study included patients with FGIDs, referred for a gastroenterologist consultation in a secondary gastroenterology practice unit. Patients were divided into three groups - patients only with IBS (IBS group), patients only with functional dyspepsia (FD) (FD group), patients with mixed symptoms IBS and FD (Mixed group). Patient levels of tissue transglutaminase IgA (tTG-IgA) and/or antiendomysial IgA group antibodies (EMA-IgA) were evaluated. Four duodenal biopsies were obtained and reported according to Marsh classification. Patients diagnosed or being referred for confirmation of CD were excluded from the study. Results: Overall, 1,833 FGIDs patients were enrolled. Celiac serology was available for 1,570 patients, duodenal histology for 582 patients, both histology and serology for 319 patients. In total, celiac seropositivity was present in $1.78 \%$ (28/1570) (3.18\% in IBS group, $0.90 \%$ in FD group and $1.11 \%$ of cases in the mixed group). Fifteen patients had histopathological changes (2.58\%; 15/582). Three IBS patients (2.36\%) were both serology and biopsy positive. None of the FD patients had CD.

Conclusion: Prevalence of biopsy-proven CD in patients from Latvia with FGIDs was low. Routine screening for CD could be considered only among patients with IBS.
\end{abstract}

Key words: celiac disease - functional gastrointestinal disorders - irritable bowel syndrome - functional dyspepsia - immunoglobulin A antibodies to tissue transglutaminase - immunoglobulin A antibodies to endomysium.

Abbreviations: CD: celiac disease; EMA-IgA: immunoglobulin A antibodies to endomysium; FD: functional dyspepsia; FGIDs: functional gastrointestinal disorders; GFD: gluten-free diet; IBS: irritable bowel syndrome; IBS-A: alternating type irritable bowel syndrome; IBS-C: constipation-predominant form of irritable bowel syndrome; IBS-D: diarrhoea-predominant form of irritable bowel syndrome; IgA: immunoglobulin A; tTGIgA: immunoglobulin A antibodies to tissue transglutaminase.

\section{INTRODUCTION}

Studies report a symptom overlap between classical celiac disease (CD) and functional gastrointestinal disorders (FGIDs), in particular, irritable bowel syndrome (IBS) and its diarrhoea-predominant form (IBS-D). Abdominal discomfort, bloating and altered stools are characteristic for both $\mathrm{CD}$ and IBS, while a number of patients with CD present with abdominal pain, nausea and vomiting characteristic for functional dyspepsia (FD) $[1,2]$. As a result, patients with CD may be misdiagnosed with IBS or FD. On the other hand, one could expect a higher proportion of CD cases among FGIDs patients than in the general population.

Several studies have addressed this issue beforehand. Sanders et al. [3] reported the prevalence of biopsy-proven CD in $4.7 \%$ of IBS patients fulfilling Rome II criteria while the prevalence of the disease was $0.67 \%$ in the control group without IBS symptoms. Ford et al. [4] estimated that the pooled prevalence of either celiac seropositivity and biopsy-proven CD among IBS patients was 1.63\% (95\% confidence interval, $0.7-3.0)$ and $4.1 \%$ (1.9-7.0), respectively.

In contrast, El-Salhy et al. [5] reported a $0.4 \%$ prevalence of biopsy-proven CD in 968 IBS patients from Norway, all of whom fulfilled the Rome III criteria for IBS-D. This author also 
recently reported that $1.4 \%$ of IBS patients (all of whom had IBS-D subtype) had another gastrointestinal organic disease, histopathologically proven. Out of these patients, $0.3 \%(5 / 1,489)$ had CD [6]. A study by Cash et al. [7] conducted in a US referral population also showed a low prevalence of biopsy-proven CD of $0.41 \%$ in 492 patients with non-constipated IBS (NC-IBS). This prevalence was similar to that observed in healthy controls $(0.44 \%, \mathrm{p}>0.99)$. Another study in the US by Choung et al. [8] reported that the prevalence of CD among subjects with IBS on the basis of serologic markers was $1 \%$ (95\% CI, 0.7\%-1.4\%) [8]. These results question the value of opportunistic screening in patients with suspected IBS in the US. However, Ford AC [9] suggested that recommendations for practice in either primary or secondary care in other countries should not change.

There is less evidence of overlap between FD and CD, although the prevalence of $\mathrm{CD}$ has been also reported double than that in the general population [10]. Ford et al. [11] have reported that the prevalence of biopsy-proven $\mathrm{CD}$ in patients with FD is $1 \%$, which is of a similar magnitude to that in the general population of some countries.

The standard initial evaluation of patients suspected of $\mathrm{CD}$ includes the detection of immunoglobulin $\mathrm{A}$ antibodies to tissue transglutaminase (tTG-IgA) or immunoglobulin A antibodies to endomysium (EMA-IgA) if tTG-IgA is weakly positive, and parallel exclusion of IgA deficiency $[12,13]$. The prevalence of the IgA deficiency is reported to be about $0.2 \%$ in the general Caucasian population $[14,15]$, and higher among CD patients [16]. Duodenal histopathology (Marsh classification) is always necessary if the antibodies are low or negative, and if there are no signs/symptoms of malabsorption. In certain situations, biopsies may be omitted. According to the European Society for Paediatric Gastroenterology, Hepatology and Nutrition (ESPGHAN) guidelines for the diagnosis of $\mathrm{CD}$ in children, a confirmatory small intestinal biopsy is no longer necessary in genetically predisposed individuals who are symptomatic and who have a tTG-IgA of at least 10 times the upper limit of normal, a positive EMA-IgA and a good clinical response to the gluten-free diet (GFD) [12]. Several studies have suggested omitting duodenal biopsies also in adult patients [17].

A recent review by Ludvigsson et al. [18] suggests that, although CD fulfils several WHO criteria for mass screening, sufficient proof of its benefit is still lacking and a strategy of proactive case-finding in high-risk groups is the appropriate management [18]. In a number of current guidelines, IBS is listed as one of the risk conditions for CD [8], the role of FD is not so clear. The objective of the current study was to analyze the prevalence of CD characteristic positive test results in the patient population with FGIDs in Latvia - one of the three Baltic States in Northern Europe. In particular, the prevalence of positive tTG-IgA, EMA-IgA and positive histology in patients with IBS and FD were sought.

\section{METHODS}

\section{Study design}

Patients with FGIDs, referred for a gastroenterologist consultation in a secondary gastroenterology practice unit of Digestive Diseases Centre GASTRO (from 2004 to 2014) were included in our retrospective study. Patients with IBS and/or FD diagnosis and available celiac tests results (tTG-IgA \pm EMA-IgA \pm histopathological findings) were included in the study. Patients with previously confirmed CD, clinically suspected CD or patients being referred to our unit for confirmation of the disease were excluded from this analysis. The diagnosis of IBS and FD was made under clinical settings by using Rome II criteria [19]; during our study, Rome III criteria were available [20]. Patients were divided into three groups: patients only with IBS (IBS group), patients only with FD (FD group), patients with mixed symptoms IBS and FD (Mixed group). Patients with IBS were grouped in types as diarrhoea predominant disease (IBS-D), constipation-predominant disease (IBS-C), and alternating type (IBS-A). The FD group was not subtyped for the analysis.

\section{Diagnostic tests}

The routine workup for CD diagnosis included serological testing and duodenal histopathology as a confirmatory test. Celiac serology included the measurement of tTG-IgA and, in parallel, total serum IgA was determined to rule out IgA deficiency. At the beginning of the study period also EMAIgA was detected, but it was not available for routine clinical practice thereafter.

TTG-IgA was detected according to laboratory standards which changed during the study period (Hycor HYTEC ${ }^{\mathrm{m}}$, Hycor Biomedical Ltd., U.K; Immulite 2000 system, Siemens Healthcare Diagnostics Inc., U.K.; Alegria, Orgentec, Germany). EMA-IgA was assessed as described previously [21]. Total IgA was measured by the immunoturbidimetric method (Roche Diagnostics) with the cut-off value below $40 \mathrm{mg} / \mathrm{dl}$ for IgA deficiency. Values below $7 \mathrm{mg} / \mathrm{dl}$ were considered to correspond to severe IgA deficiency. Celiac disease seropositivity was considered if either tTG-IgA and/ or EMA-IgA was positive.

Four duodenal and one bulbus biopsy were obtained during the upper endoscopy for histopathological examination. Histopathology was reported according to Marsh classification as modified by Oberhuber. On separate occasions, when celiac disease was suspected, the immunohistochemical stain was employed for the detection of CD3 intraepithelial lymphocytes. Only expert pathologist reports were considered.

Criteria for CD were positive serology (either tTG-IgA and/or EMA-IgA) according to the manufacturer and positive histopathology (Marsh III lesions were considered to be diagnostic for the disease) [22].

\section{Statistical analysis}

Descriptive statistics were used to characterize the study group and the proportion of the positive test results according to the predefined criteria. Mean values, range, standard deviation (SD) and 95\% confidence intervals (CI) of means were used to describe the distribution of the measurement results. Groups were analyzed using $\mathrm{n} \times \mathrm{n}$ tables and Chisquare test and Fisher's exact test (acceptable alpha <0.05). The analysis included different families of hypothesis tests. In families with more than one hypothesis (e.g. difference among IBS, FD and overlapping groups), Bonferroni correction was applied (alpha for statistical significance in the mentioned 3 groups case was decreased to $0.05 / 3=0.0167$ ). 


\section{Ethical considerations}

The study protocol was approved by the Committee of Ethics at the Institute of Experimental and Clinical Medicine of the University of Latvia. This was a retrospective study, therefore no consent form was applicable.

\section{RESULTS}

\section{Total patient sample}

Altogether 1,833 patients with FGIDs [1,195 (65.19\%) women, median age 37 years (range 18-83)] were included in the study. In total, FD was diagnosed in 1,165 patients, IBS in 1,177 patients. Further, 656 patients were included in the FD group, 668 patients in the IBS group, 509 patients in the Mixed group. In the IBS group, 314 patients $(47.01 \%)$ had IBS-D, 127 patients (19.01\%) had IBS-C and 227 patients (33.98\%) had IBS-A.

The serology for CD was available for 1,570 patients ( $\mathrm{tTG}$ IgA was done in 1561 patients, EMA-IgA in 141 patients; both the tests were performed in 132 cases). Detection of IgA was conducted in 1,234 patients. Duodenal biopsies were available from 582 patients, additional bulbus biopsies from 70 patients. Both histology and serology were available for 319 patients (Fig. 1).

\section{Serologic findings}

Within the entire group, celiac seropositivity (either tTGIgA and/or EMA-IgA positive) was $1.78 \%(28 / 1,570)$. The prevalence of tTG-IgA positivity was $1.79 \%(28 / 1,561 ; 95 \% \mathrm{CI}$ : $2-2.6)$, and the prevalence of EMA-IgA positivity was $1.42 \%$ (2/141; 95\%CI: 0.2-5). Three patients were diagnosed with IgA deficit $(0.24 \%, 95 \% \mathrm{CI}: 0.01-0.7)$; out of them, one had severe IgA deficiency $(0.08 \%, 95 \% \mathrm{CI}: 0.001-0.5)$. None of the IgA deficient patients had undergone duodenal biopsies.

In the Mixed group serology (TTG-IgA and/or EMA-IgA) was positive in $1.11 \%$ of the cases $(5 / 451 ; 95 \% \mathrm{CI}$ : $0.4-2.6)$, in the IBS group in $3.18 \%$ of the cases (18/566; 95\% CI: $0.9-5)$ and in the FD group in $0.9 \%$ of the cases $(5 / 553$; 95\%CI: $0.3-2.1)$ ( $\mathrm{p}=0.007$ if all three groups are compared). The IBS group had positive serology more often than the FD group $(\mathrm{p}=0.0073)$ and the Mixed group ( $p=0.0273$ ), although the result in the second comparison was not statistically significant after the Bonferroni correction. There was no difference in seropositivity between the Mixed group and the FD group ( $\mathrm{p}=0.7456)$.

Among the patients in the IBS group, this test positivity rate was $3.68 \%(10 / 272,95 \% \mathrm{CI}: 4-5.9)$ in the IBS-D group, $1.9 \%$ (2/105; 95\%CI: 0.7-4.5) in the IBS-C group, $3.17 \%$ (6/189; 95\%CI: 0.7-5.7) in the IBS-A group, but the difference was not statistically significant $(\mathrm{p}=0.6941)$.

\section{Histological findings}

In almost half of the patients with a positive serology test (46.43\%; 13/28; 95\%CI: 27.5-66.1) duodenal mucosa histopathology examination was available. Most of the seropositive cases (76.9\%; 10/13; 95\%CI: 46.2-95) were found to be biopsy negative for $\mathrm{CD}$, although results were only slightly positive (tTG-IgA level was $8.1-22.8 \mathrm{U} / \mathrm{ml}$ ).

Only 15 patients had histopathological changes $(2.58 \%$; 15/582; 95\%CI: 4-4.2\%), most of them with Marsh I lesions (1.89\%; 95\%CI: 0.9-3.4); Marsh III lesions were found only in 4 cases $(0.69 \%$ of the biopsies investigated, 95\%CI: $0.2-$ $7 \%)$. One patient with a positive biopsy (Marsh IIIA) had

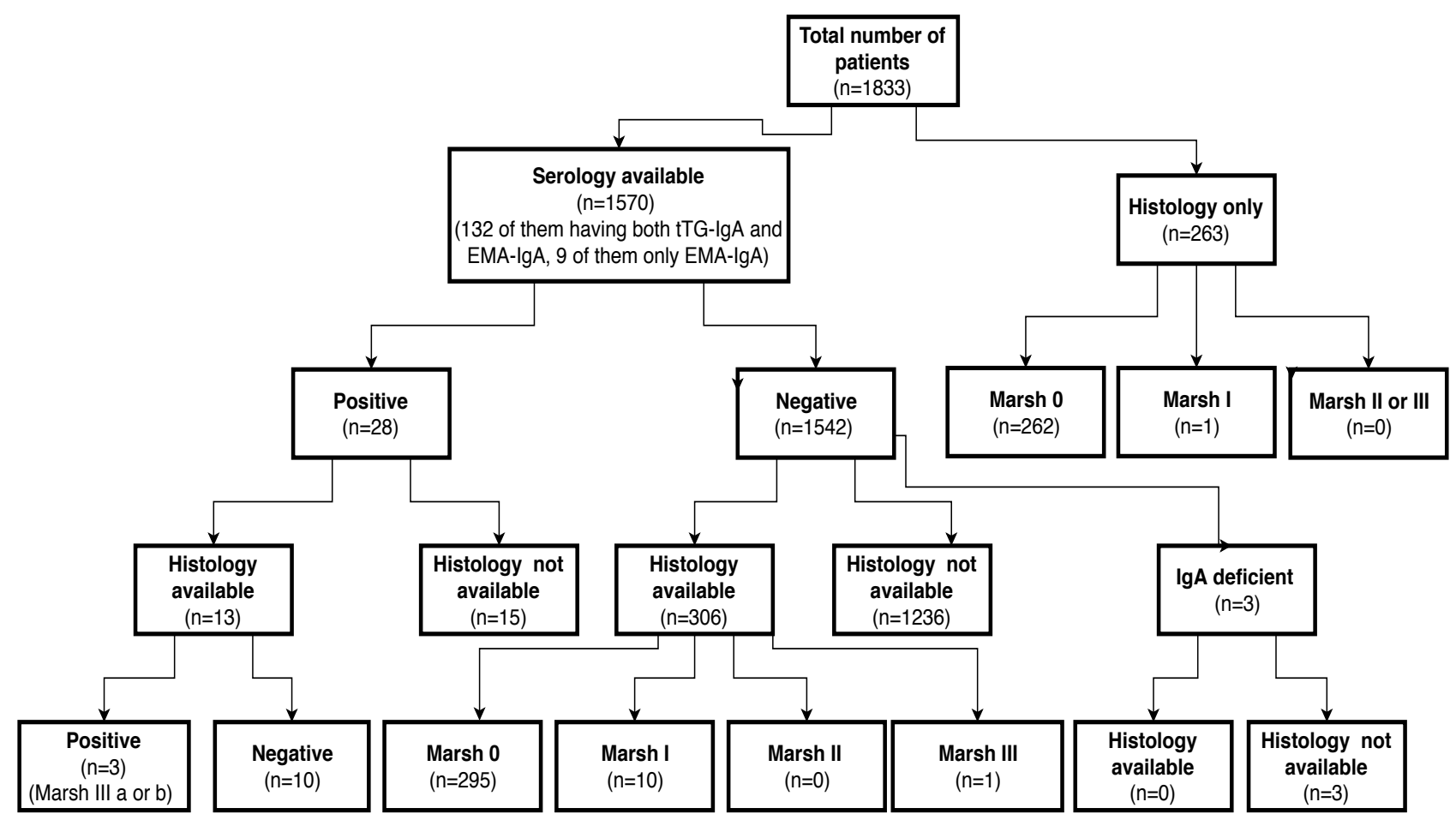

Fig. 1. Flowchart of sample characteristics and findings. tTG-IgA: immunoglobulin A antibodies to tissue transglutaminase; EMA-IgA: immunoglobulin A antibodies to endomysium. 
negative serological markers (repeatedly detected) for $\mathrm{CD}$, IgA was normal. HLA-DQ analysis was not available. Three IBS patients were both serology and biopsy positive $(0.94 \%$ of FGIDs patients, $95 \% \mathrm{CI}: 0.2-2.7 \%$, and $1.37 \%$ of IBS patients (IBS in total group), 95\%CI: $0.3-4$ ) and $2.36 \%$ of IBS patients (95\%CI: 0.81-6.71) (IBS only group). Two of them were IBS-D patients (3.08\%; 2/65 IBS-D patients; 95\%CI: 1-7.3), one was IBS-A patient (2.94\%; 1/34 IBS-A patients; 95\%CI: 2.7-8.6). None of the FD patients had serologically and histologically proven CD (Fig. 2).

To sum up, prevalence of positive celiac serology and biopsy-proven diagnosis was higher in the group of IBS-D patients (positive tTG-IgA and/or EMA-IgA 3.68\%, 10/272; biopsy-proven diagnosis 3.08\%, 2/65) and also IBS-A patients (positive tTG-IgA and/or EMA-IgA 3.17\%, 6/189; biopsyproven diagnosis $2.94 \%, 1 / 34$ ). No biopsy-proven CD patients were found in the group of IBS-C patients. Positive serological tests were found in 2 (1.90\%) of 105 patients in this group.

Prevalence of biopsy-proven CD in subjects with FGIDs was $0.94 \%$ (3/319, 95\%CI: 0.2-2.7). Prevalence of the disease among the patients from the IBS group was $2.36 \%(3 / 127$, 95\%CI: 0.3-5).

\section{DISCUSSION}

This study represents the first data on the prevalence of CD among a large sample of patients with FGIDs from Latvia. Our study population in respect to FGIDs was quite typical for Europe. There is a substantial overlap between IBS and FD varying from $15 \%$ to $42 \%$, depending on the diagnostic criteria used for each [23]. Substantial overlap between the two conditions was also detected in our study: $27.77 \%(509 / 1,833)$.

Previously the prevalence of CD in dyspeptic patients has been reported to be higher than that in controls [10,
24]. However, a meta-analysis done by Ford et al. [11] demonstrated that prevalence of biopsy-proven CD among dyspeptic patients was only $1 \%$, which was similar to that in the general population of some countries, suggesting that subjects with FD were not more likely to harbour undiagnosed $\mathrm{CD}$ than these individuals. This corresponds with our data, showing no seropositive biopsy-proven CD case among FD patients.

However, another meta-analysis by Ford et al. [4], addressing the prevalence of CD among IBS patients, demonstrated that the pooled prevalence of serologically (either positive EMA-IgA or tTG-IgA) and biopsy-proven CD was $1.63 \%$ (95\%CI: $0.7-3.0)$ and $4.1 \%$ (95\%CI: $1.9-7.0)$, respectively. Therefore, in contrast to dyspeptic patients, the odds for biopsy-proven CD in individuals with IBS were more than 4-fold higher than that in healthy controls [4]. In our study group, CD seropositivity among IBS patients was also significantly higher compared to seropositivity among FD patients $(3.18 \%(18 / 566)$ vs $0.9 \%$ (5/553), respectively). In addition, all cases of $\mathrm{CD}$ in our study group were among IBS patients; therefore, the total prevalence of CD among IBS patients (taking into account only IBS patients with available serology and histology data) was $2.36 \%$ (3/127).

Since IgA deficit was not common in our study group (0.24\% that corresponds to data from healthy Caucasians $[14,15])$ and none of the $\operatorname{IgA}$ deficit patients had a positive biopsy, IgA deficiency could not be the reason for the low prevalence of CD in FGIDs patients.

Evaluating data from nearby countries, in Poland CD prevalence among IBS-D patients was high: 7\% (serological and histological CD detection) [25] or $12.5 \%$ among IBS patients regardless of the subtype (serological and genetical CD detection) [26]. At the same time, low prevalence $(0.3 \%$ and $0.4 \%$ ) of the biopsy-proven CD was detected in IBS patients

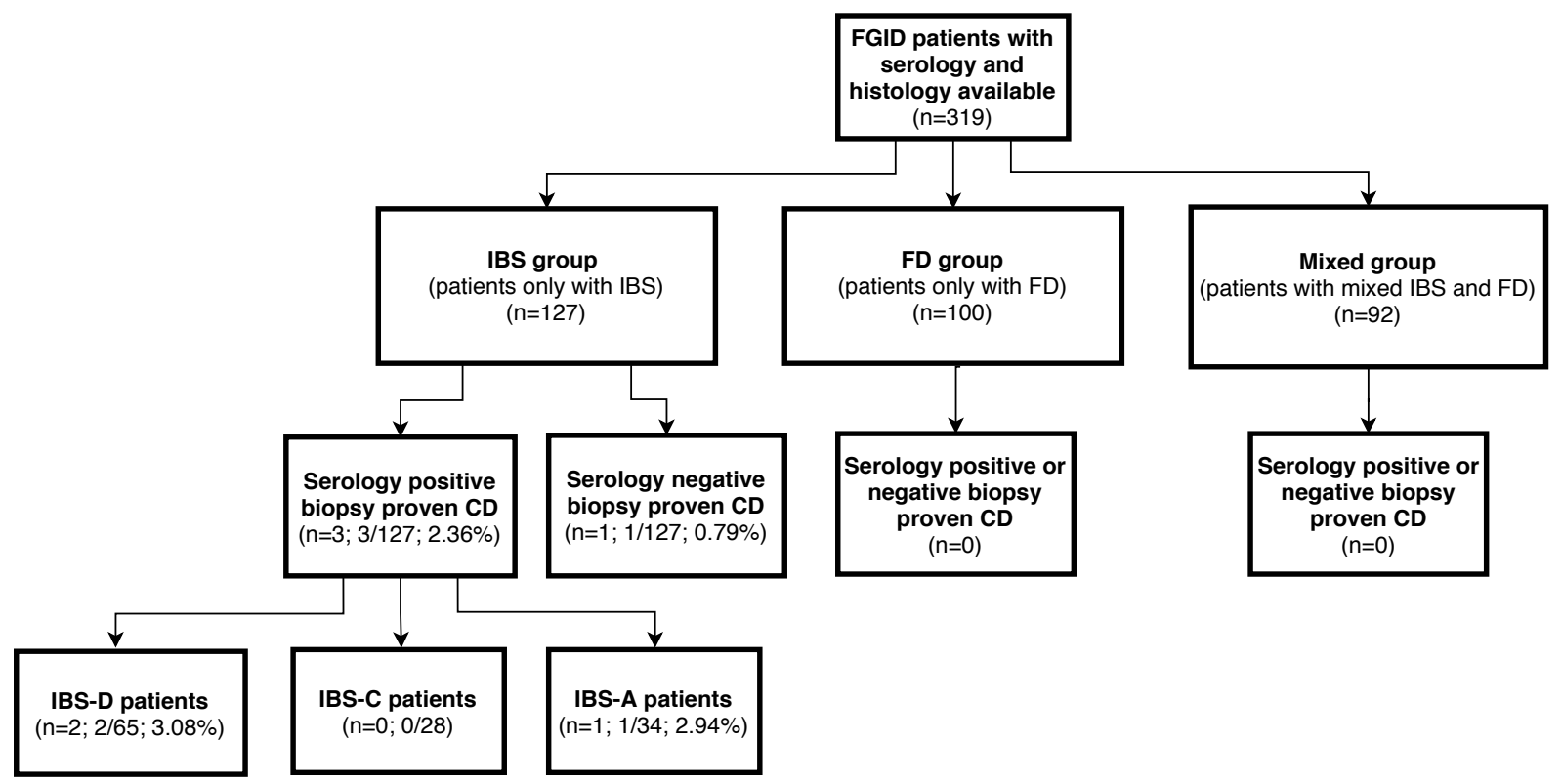

Fig. 2. Flowchart of patients with functional gastrointestinal disease in relation to the presence of celiac disease (in patients with both serology and histology available). FGIDs: functional gastrointestinal disorders; IBS: irritable bowel syndrome; FD: functional dyspepsia; IBS-D: diarrhoea-predominant form of irritable bowel syndrome; IBS-C: constipation-predominant form of irritable bowel syndrome; IBS-A: alternating type irritable bowel syndrome. 
from a high CD prevalence country Norway, all of whom fulfilled the Rome III criteria for IBS-D $[5,6]$.

Our previous study suggested that the prevalence of CD in Latvian adults was lower than in neighboring Nordic countries [27]. Prevalence of CD in Latvia based on sero-genetic testing approach ranged from 0.35 to $0.49 \%$ depending on the criteria used (ELISA, CIA, EMA-IgA and DQ 2.5/8). We have to mention that unfortunately, EMA-IgA positivity was not comparable between the present and the previous study, because a different methodology was used. Nevertheless, the prevalence of the tTG-IgA positivity in patients with FGIDs approximated the prevalence in the general population $(1.79 \%$, 95CI: $1.21-2.61$ and $1.66 \%, 95 \mathrm{CI}: 1.12-2.46)$. If population data are compared with data of patient subgroups in our study (Mixed group, FD group, IBS group, IBS-D, IBS-C, IBS-A), significantly higher prevalence of tTG-IgA positivity was observed only in the IBS group (positive tTG-IgA in $1.66 \%$ vs $3.18 \%$ cases; $\mathrm{p}=0.0321$ ).

Although previously mentioned meta-analysis by Ford et al. [4] has been addressing the possible differences in the prevalence of CD among IBS patients between the United States, Europe, and the Middle East (and no statistically significant differences were detected in OR for a positive EMAIgA or tTG-IgA result between these areas [4]), the prevalence of $\mathrm{CD}$ in the general population differs. For example, the prevalence of CD in Europe and North America is generally considered to be approximately $0.7-1.5 \%$ [27]. A recent multinational study in Europe found big differences in $\mathrm{CD}$ prevalence, with the lowest prevalence $(0.3 \%)$ in Germany and the highest in Finland (2.4\%), despite using common criteria for CD diagnosis [18]. Countries with a high prevalence include also Sweden and Great Britain [27]. Such potential differences were not considered in the meta-analysis by Ford et al. [4]. Furthermore, the European case-control studies available for the OR analysis originated mainly from high CD prevalence countries (Sweden and UK); in the prevalence analysis of test positivity among IBS patients, our study has been the only one which originated in Baltic countries. Therefore, considering our results and comparatively lower CD positivity in Latvia, the data presented in the meta-analysis by Ford et al. [4] could be an overestimate for Europe as a total, since limited data originated from the Baltic countries, and this part of Europe might have a lower prevalence of CD. The data regarding CD prevalence among patients and in the population are important in order to answer the question about the possible introduction of CD screening.

It has been estimated that screening for CD could be costeffective when the prevalence of $\mathrm{CD}$ in a specific population is $1 \%$ or greater $[12,28]$. As mentioned before, Ford et al. [11] have reported that the prevalence of biopsy-proven CD in patients with dyspepsia is $1 \%$ similar to that of the general population, and therefore systematic screening for CD would not be recommended based on disease prevalence alone. Since none of the FD patients in our study was positive for $\mathrm{CD}$, screening for CD among FD patients would not be reasonable also in our population.

However, several guidelines have suggested that screening for CD would be recommended for people at an increased risk for $\mathrm{CD}$ (like individuals with first-degree relatives with
$\mathrm{CD}$, with type 1 diabetes, autoimmune thyroid disease, or autoimmune liver disease $[29,30])$. IBS is listed as one of the risk conditions for $\mathrm{CD}$ in a number of current guidelines. For example, the World Gastroenterology Organization suggests that a low threshold for investigation should be maintained in high-prevalence regions (those with prevalence more than $1 \%$ in the general population) [12]. Cost-effectiveness data by Spiegel et al. [28] revealed that testing for CD was likely to be cost-effective in IBS-D cohorts if the prevalence was above $1 \%$ and was the dominant strategy when the prevalence exceeded 8\% [28]. Although the American College of Gastroenterology position statement does not recommend investigation in patients with symptoms of IBS unless alarming symptoms are present; testing for CD should be done in patients with IBS-D or IBS-A [31].

The routine exclusion of CD in all patients with IBS without differentiating between the subtypes is recommended by the National Institute for Health and Clinical Excellence guidelines in the United Kingdom [32].

In our study, the IBS-D group had also higher seropositivity (3.68\%) compared to IBS-A (3.17\%) and IBS-C (1.90\%), but the difference was not statistically significant. However, among three seropositive and histologically proved cases, two were from IBS-D group (3.08\%; 2 of 65 IBS-D patients) and one from IBS-A group (2.94\%; 1 of 34 IBS-A patients). Since in our study sample prevalence of CD among IBS patients was $2.36 \%$, screening could be considered only among IBS patients.

There is increasing evidence that among patients with high tTG-IgA levels (at least 10 times the upper limit of normal) a small intestinal biopsy is not needed to confirm the diagnosis of CD. Such an approach has also been recommended by the ESPGHAN guidelines for the diagnosis of CD in the pediatric population [12], because a large number of studies conducted in a pediatric or mixed adult/pediatric population showed that high tTG-IgA levels $(\geq 100 \mathrm{U} / \mathrm{mL})$ are associated with histological lesions compatible with CD [33]. Similarly, in our study group all three patients with tTG-IgA $\geq 100 \mathrm{U} / \mathrm{mL}$ had Marsh III histopathology in biopsy samples. Worth mentioning is the fact that there was a high prevalence of positive serology with normal histology in our study, although the results were only slightly positive (tTG-IgA level was $8.1-22.8 \mathrm{U} / \mathrm{ml}$ ). These patients might have either a false-positive test or a form of CD that has not yet determined significant mucosal injury (potent CD) [34]. Vecchi et al. [35] noted a high prevalence of false-positive tTG-IgA results in a group of patients with chronic liver disease [35]. Also type 1 diabetes patients may have elevated tTG-IgA levels, which may spontaneously normalize [36].

The main limitation of our study is its retrospective character. Because of that, there was no possibility to investigate the effect of CD on the incidence of FGIDs. Further prospective cohort studies are necessary to investigate these relationships. Another limitation is the number of individuals with the availability of both serological and histological results for diagnosis of CD $(n=319)$. Further, IgA was not detected for all patients; therefore, there might have been false-negative serology in some cases. Nevertheless, the total number of studied FGIDs patients is high and data were available for the majority of patients $(67.3 \%$ of the total study population and 
$81 \%$ of 319 patients with both serology and histology available had IgA results). Further, during our study period methods for tTG-IgA detection had changed; therefore, results were interpreted according to laboratory standards.

Treatment for FGIDs can be a clinical challenge and dyspepsia as a symptom of CD will readily respond to a GFD. On the other hand, GFD will also show a placebo effect in individuals without $\mathrm{CD}$, thus causing confusion. Therefore, CD could be considered in dyspeptic patients to decrease the amount of unnecessary introduction of GFD, as well as a delayed diagnosis of CD.

\section{CONCLUSIONS}

Prevalence of biopsy-proven CD in patients with FGIDs from Latvia was low and tTG-IgA seropositivity did not differ among FGIDs patients and the general population. However, seropositivity of CD was significantly higher among IBS patients compared to the seropositivity in the general population (3.18\% vs. $1.66 \%)$. Since the prevalence of biopsyproven CD among IBS is $2.36 \%$, thus exceeding $1 \%$ border of cost-effectiveness for routine screening, we suggest that it could be considered among patients with IBS. Prevalence of CD and possible screening should be further studied in patients with different IBS subtypes.

Acknowledgements: The infrastructure of Digestive Diseases Centre GASTRO (Riga, Latvia) was used for the data collection. The authors would like to thank nurse Irena Sitikova for the data collection.

\section{Conflicts of interests: None to declare.}

Authors' contribution: M.L.: principal design of the study, analysis of the results, writing of the manuscript; A.D., I.D., I.K.: participation in the design of the study, analysis of the results, writing the manuscript, A.D., S.P.: data collection, I.P., I.K.: statistical analyses, L.Z., V.P., I.T., I.R., S.B., A.V.: design of the study, clinical data collection. All authors critically revised the manuscript, approved the final version to be published, and agreed to be accountable for all aspects of the work.

\section{REFERENCES}

1. Zipser RD, Patel S, Yahya KZ, Baisch DW, Monarch E. Presentations of adult celiac disease in a nationwide patient support group. Dig Dis Sci 2003;48:761-764. doi:10.1023/a:1022897028030

2. Longstreth GF, Thompson WG, Chey WD, Houghton LA, Mearin F, Spiller RC. Functional bowel disorders. Gastroenterology 2006;130 (5):1480-1490. doi:10.1053/j.gastro.2005.11.061

3. Sanders DS, Carter MJ, Hurlstone DP, et al. Association of adult coeliac disease with irritable bowel syndrome: a case-control study in patients fulfilling ROME II criteria referred to secondary care. Lancet 2001;358:1504-1508. doi:10.1016/S0140-6736(01)06581-3

4. Ford AC, Chey WD, Talley NJ, Malhotra A, Spiegel BM, Moayyedi P. Yield of diagnostic tests for celiac disease in individuals with symptoms suggestive of irritable bowel syndrome: systematic review and meta-analysis. Arch Intern Med 2009;169:651-658. doi:10.1001/ archinternmed.2009.22
5. El-Salhy M, Lomholt-Beck B, Gundersen D. The prevalence of celiac disease in patients with irritable bowel syndrome. Mol Med Rep 2011;4:403-405. doi:10.3892/mmr.2011.466

6. El-Salhy M, Gilja OH, Hatlebakk JG. Overlapping of irritable bowel syndrome with erosive esophagitis and the performance of Rome criteria in diagnosing IBS in a clinical setting. Mol Med Rep 2019;20:787-794. doi:10.3892/mmr.2019.10284

7. Cash BD, Rubenstein JH, Young PE, et al. The prevalence of celiac disease among patients with nonconstipated irritable bowel syndrome is similar to controls. Gastroenterology 2011;141:1187-1193. doi:10.1053/j. gastro.2011.06.084

8. Choung RS, Rubio-Tapia A, Lahr BD, et al. Evidence Against Routine Testing of Patients With Functional Gastrointestinal Disorders for Celiac Disease: A Population-based Study. Clin Gastroenterol Hepatol 2015;13:1937-1943. doi:10.1016/j.cgh.2015.05.014

9. Ford AC. Is it Time to Rethink Screening of Individuals With Symtoms of Irritable Bowel Syndrome for Celiac Disease? Clin Gastroenterol Hepatol 2015;13:1944-1945. doi:10.1016/j.cgh.2015.07.039

10. Bardella MT, Minoli G, Ravizza D, et al. Increased prevalence of celiac disease in patients with dyspepsia. Arch Intern Med 2000;160:14891491. doi:10.1001/archinte.160.10.1489

11. Ford AC, Ching E, Moayyedi P. Meta-analysis: yield of diagnostic tests for coeliac disease in dyspepsia. Aliment Pharmacol Ther 2009;30:28-36. doi:10.1111/j.1365-2036.2009.04008.x

12. Bai JC, Ciacci C, Corraza GR, et al. World Gastroenterology Organisation Practice Guidelines: Celiac Disease World Gastroenterology Organisation 2016. Available at: http://www.worldgastroenterology. org/UserFiles/file/guidelines/celiac-disease-english-2016.pdf

13. Downey L, Houten R, Murch S, Longson D, Guideline Development G. Recognition, assessment, and management of coeliac disease: summary of updated NICE guidance. BMJ 2015;351:h4513. doi:10.1136/bmj. h4513

14. Weber-Mzell D, Kotanko P, Hauer AC, et al. Gender, age and seasonal effects on IgA deficiency: a study of 7293 Caucasians. Eur J Clin Invest 2004;34:224-228. doi:10.1111/j.1365-2362.2004.01311.x

15. Litzman J, Sevcikova I, Stikarovska D, Pikulova Z, Pazdirkova A, Lokaj J. IgA deficiency in Czech healthy individuals and selected patient groups. Int Arch Allergy Immunol 2000;123:177-180. doi:10.1159/000024438

16. Collin P, Maki M, Keyrilainen O, Hallstrom O, Reunala T, Pasternack A. Selective IgA deficiency and coeliac disease. Scand J Gastroenterol 1992;27:367-370. doi:10.3109/00365529209000089

17. Hill PG, Holmes GK. Coeliac disease: a biopsy is not always necessary for diagnosis. Aliment Pharmacol Ther 2008;27:572-577. doi:10.1111/ j.1365-2036.2008.03609.x

18. Ludvigsson JF, Card TR, Kaukinen K, et al. Screening for celiac disease in the general population and in high-risk groups. United European Gastroenterol J 2015;3:106-120. doi:10.1177/2050640614561668

19. Talley NJ, Stanghellini V, Heading RC, Koch KL, Malagelada JR, Tytgat GN. Functional gastroduodenal disorders. Gut 1999;45(Suppl 2):II37II42. doi:10.1136/gut.45.2008.ii37

20. Drossman DA. The functional gastrointestinal disorders and the Rome III process. Gastroenterology 2006;130:1377-1390. doi:10.1053/j. gastro.2006.03.008

21. Leja M, Kojalo U, Frickauss G, Bandere B, Gavars D, Boka V. Changing patterns of serological testing for celiac disease in Latvia. J Gastrointestin Liver Dis 2011;20:121-126.

22. Bao F, Green PH, Bhagat G. An update on celiac disease histopathology and the road ahead. Arch Pathol Lab Med 2012;136:735-745. doi:10.5858/arpa.2011-0572-RA 
23. Ford AC, Marwaha A, Lim A, Moayyedi P. Systematic Review and Meta-Analysis of the Prevalence of Irritable Bowel Syndrome in Individuals with Dyspepsia. Clin Gastroenterol Hepatol 2010;8:401-409. doi:10.1016/j.cgh.2009.07.020

24. Ozaslan E, Akkorlu S, Eskioglu E, Kayhan B. Prevalence of silent celiac disease in patients with dyspepsia. Dig Dis Sci 2007;52:692-697. doi:10.1007/s10620-006-9453-1

25. Zwolinska-Wcislo M, Galicka-Latala D, Rozpondek P, Rudnicka-Sosin L, Mach T. Frequency of celiac disease and irritable bowel syndrome coexistance and its influence on the disease course. Przegl Lek 2009;66:126-129.

26. Domzal-Magrowska D, Kowalski MK, Szczesniak P, Bulska M, Orszulak-Michalak D, Malecka-Panas E. The prevalence of celiac disease in patients with irritable bowel syndrome and its subtypes. Prz Gastroenterol 2016;11:276-281. doi:10.5114/pg.2016.57941

27. Leja M, Shums Z, Nikitina-Zake L, et al. Prevalence estimation of celiac disease in the general adult population of Latvia using serology and HLA genotyping. United European Gastroenterol J 2015;3:190-199. doi:10.1177/2050640615569379

28. Spiegel BM, DeRosa VP, Gralnek IM, Wang V, Dulai GS. Testing for celiac sprue in irritable bowel syndrome with predominant diarrhea: a cost-effectiveness analysis. Gastroenterology 2004;126:1721-1732. doi:10.1053/j.gastro.2004.03.012

29. Murch S, Jenkins H, Auth M, et al. Joint BSPGHAN and Coeliac UK guidelines for the diagnosis and management of coeliac disease in children. Arch Dis Child 2013;98:806-811. doi:10.1136/archdischild-2013-303996
30. Ludvigsson JF, Rubio-Tapia A, van Dyke CT, et al. Increasing incidence of celiac disease in a North American population. Am J Gastroenterol 2013;108:818-824. doi:10.1038/ajg.2013.60

31. American College of Gastroenterology Task Force on Irritable Bowel Syndrome, Brandt LJ, Chey WD, et al. An evidence-based position statement on the management of irritable bowel syndrome. Am J Gastroenterol 2009;104(Suppl 1):S1-S35.

32. National Institute for Health and Care Excellence. Coeliac disease recognition, assessment and management. 2015. Available at: https:// www.nice.org.uk/guidance/ng20

33. Mubarak A, Wolters VM, Gmelig-Meyling FH, Ten Kate FJ, Houwen RH. Tissue transglutaminase levels above $100 \mathrm{U} / \mathrm{mL}$ and celiac disease: a prospective study. World J Gastroenterol 2012;18:4399-4403. doi:10.3748/wjg.v18.i32.4399

34. Castellaneta S, Piccinno E, Oliva M, et al. High Rate of Spontaneous Normalization of Celiac Serology in a Cohort of 446 Children With Type 1 Diabetes: A Prospective Study. Diabetes Care 2015;38:760-766. doi:10.2337/dc14-2890

35. Vecchi M, Folli C, Donato MF, Formenti S, Arosio E, de Franchis R High rate of positive anti-tissue transglutaminase antibodies in chronic liver disease. Role of liver decompensation and of the antigen source. Scand J Gastroenterol 2003;38:50-54.

36. Rinawi F, Badarneh B, Tanous O, Bashir H, Tennenbaum-Rakover Y, Peleg S. Elevated anti-tissue transglutaminase antibodies in children newly diagnosed with type 1 diabetes do not always indicate coeliac disease. Acta Paediatr 2019;108:149-153. doi:10.1111/apa.14398 